\begin{tabular}{|c|c|c|}
\hline Beitr. Ent. & Keltern & ISSN 0005-805X \\
\hline $\mathbf{5 6}(2006) 2$ & S. $281-296$ & 15.12 .2006 \\
\hline
\end{tabular}

\title{
On the Italian species of Drusilla LEACH, 1819, with a note on D. taygetana Assing
}

\section{(Coleoptera: Staphylinidae, Aleocharinae)}

With 55 figures and 2 maps

Volker Assing

Summary

The Italian representatives of the aleocharine genus Drusilla LEACH, 1819 are revised. Three species are recognised; the presence of a fourth species, D. erichsoni (Peyron, 1857), in Italy is doubtful. The enormous variation of external characters and the spermatheca of D. italica (Bernhauer, 1903) is described, illustrated, and discussed. It is interpreted as clinal rather than interspecific or intersubspecific variation. Based on examined records, the distributions are mapped. A key to species is provided. The previously unknown male sexual characters of D. taygetana Assing, 2005 are described and illustrated.

Keywords

Coleoptera, Staphylinidae, Aleocharinae, Drusilla, Italy, Greece, taxonomy, new records, key to species, distribution

\section{Zusammenfassung}

Die in Italien vertretenen Arten der Gattung Drusilla Leach, 1819 werden revidiert. Drei Arten werden nachgewiesen; das Vorkommen einer vierten Art, D. erichsoni (Peyron, 1857), in Italien ist zweifelhaft. Die enorme Variabilität äußerer Merkmale sowie der Spermatheca von D. italica (Bernhauer, 1903) wird beschrieben, abgebildet und diskutiert. Offenbar handelt es sich um klinale - nicht interspezifische oder intersubspezifische - Variabilität. Für die drei in Italien sicher nachgewiesenen Arten werden Verbreitungskarten und eine Bestimmungstabelle, die auch D. erichsoni berücksichtigt, erstellt. Die bisher unbekannten männlichen Sexualmerkmale von D. taygetana Assing, 2005 werden beschrieben und abgebildet.

\section{Introduction}

According to recent revisions (Assing 2005a, 2005b), the genus Drusilla LeAch of the Lomechusini is represented in the Western Palaearctic region - including Middle Asia - by 29 species in two subgenera. Three species were previously confirmed to occur in Italy (Assing 2005b, ZanetTi 1995): the Palaearctic D. canaliculata (FABricius), D. memnonia (Märkel) from Sicily and North Africa, and the Italian endemic D. italica 
(Bernhauer). Luigioni (1929) reported D. italica also from the Cottian Alps in northwestern Italy. Since $D$. italica is confined to southern Italy and the name was at that time believed to be synonymous with D. erichsoni (PeYron) (see Porta 1926), this would suggest that the latter may be present on Italian territory, too. However, this record requires verification.

A systematic study of the representatives of the Eastern Mediterranean (Greece, southern Turkey, Middle East) revealed that a reliable separation of Drusilla species is often difficult, not only owing to considerable intraspecific variation especially of external characters, of secondary sexual characters, and to some extent of the shape of the spermatheca, but also to weakly pronounced interspecific character divergence. Remarkably, in contrast to most other genera of Aleocharinae, the morphology of the aedeagus is often of little taxonomic use, since it tends to be uniform and consequently provides weakly pronounced differences even among more distantly related taxa (Assing 2005b). HLAváč (2005) observed a similar situation also for Lomechusa GravenHorst, 1806, another genus of Lomechusini. In contrast to Lomechusa, however, the shape of the spermatheca was found to be of high taxonomic significance in Drusilla and is often the most reliable character for an identification at the species level (Assing 2005b).

The present study was initiated by material of $D$. italica made available to me by Fernando Angelini, Francavilla Fontana. The pronounced variation of external characters such as coloration, microsculpture, size, pronotum shape, secondary sexual characters, and the made me suspect that material previously identified as D. italica was in fact a complex of different species or subspecies. Subsequently, additional material from various public and private collections was studied in order to clarify the taxonomic status and the zoogeography of Italian Drusilla and of D. italica in particular.

\section{Material, measurements, and maps}

The material examined is deposited in the following collections:

$\begin{array}{ll}\text { DEI } & \text { Deutsches Entomologisches Institut, Müncheberg (L. Zerche) } \\ \text { FMNH } & \text { Field Museum of Natural History, Chicago (J. Boone) } \\ \text { MCSNV } & \text { Museo Civico di Storia Naturale Verona (L. Latella) } \\ \text { NHMW } & \text { Naturhistorisches Museum Wien (H. Schillhammer) } \\ \text { cAng } & \text { private collection F. Angelini, Francavilla Fontana } \\ \text { cAss } & \text { author's private collection } \\ \text { cSch } & \text { private collection M. Schülke, Berlin } \\ \text { cZan } & \text { private collection A. Zanetti, Verona }\end{array}$

Head length was measured from the anterior margin of the clypeus to the posterior constriction, elytral length from the apex of the scutellum to the posterior margin of the elytra.

The maps were generated using the online generic mapping tool (GMT) of the Geomar website at www. aquarius.geomar.de/omc. 


\section{Drusilla canaliculata (FABRICIUS, 1787) (Figs 1-6, Map 1)}

\section{Types examined}

Astilbus leonii Porta: Syntypes: $10^{\star}, 3$ o 9 : canaliculatus v. Leonii Porta, Cerchio, Leoni / Syntypus / D.E.I. v. Heyden / var. Leonii Porta / Drusilla canaliculata (Fabricius) det. V. Assing 2006 (DEI).
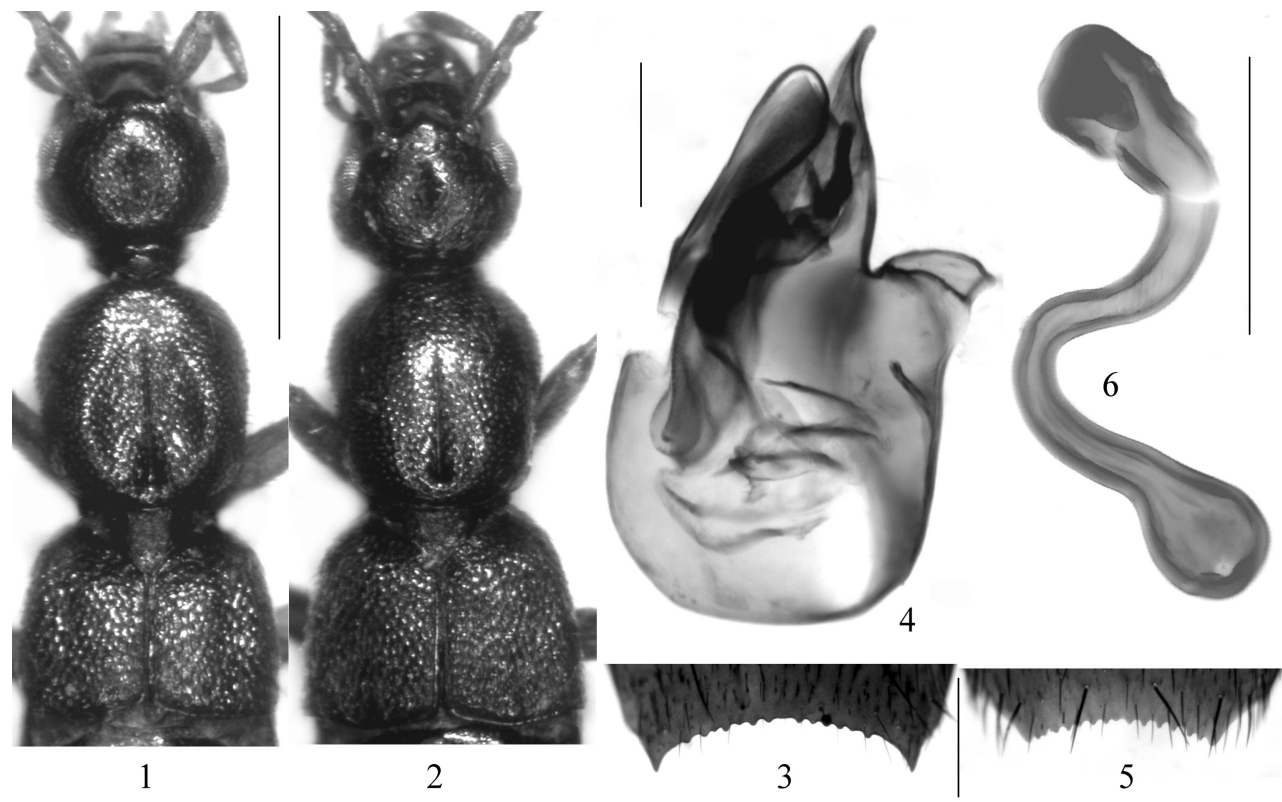

Figs 1-6: Drusilla canaliculata: o forebody (1); of forebody (2); posterior margin of o tergite VIII (3); median lobe of aedeagus in lateral view (4); posterior margin of 9 tergite VIII (5); spermatheca (6). Scale bars: 1-2: $1.0 \mathrm{~mm}$; 2-6: $0.2 \mathrm{~mm}$.

Additional material examined: Trentino-Alto Adige: 1 ex., Riva/Garda, Lago di Loppio, 22.VI.1989, leg. Wunderle (cWun). 1 ex., Lago di Tenno, N Riva, 560 m, 2.VIII.1994, leg. Zerche (DEI); 1 ex., Lago di Varna (Bressanone-BZ), 17.VII.1971, leg. Zanetti (cZan); 1 ex., Bressanone (BZ), Tiles, 30.VII.1973, leg. Sette (cZan); 1 ex., Lago di Caldaro (BZ), 21.IV.1992, leg. Zanetti (cZan); 1 ex., Smarano, Preddia, 1300 m, 27.VII.1970, leg. Zanetti (cZan); 1 ex., Denno (TN), Sabino, 21.VIII.1973, leg. Zanetti (cZan); 1 ex., Coredo (TN), Laghi Palu, 21.IV.1987, leg. Zanetti (cZan); 13 exs., Castel Thun (TN), VIII-IX.2000, leg. Zanetti (cZan); 4 exs., V. di Rabbi (TN), Saent, 1800 m, leg. Zanetti (cZan); 1 ex., Sega di Ala (TN), 2.VI.1986, leg. Zanetti (cZan); 1 ex., Smarano (TN), 1000 m, 2.VIII.1974, leg. Zanetti (cZan); 1 ex., Levico (TN), Ontano, 26.X.1989, leg. Zanetti (cZan); 3 exs., Rovereto (TN) (MCSNV); 1 ex., Cles (TN), Mechel, 1.V.1989, leg. Zanetti (cZan); 1 ex., Lagorai (TN), Val Ilba, 1800 m, 19.VI.1994, leg. Zanetti (cZan); 3 exs., Carisolo, 800 m, 12.IV.1971, leg. Cornacchia (cZan); 16 exs., Lodrone, 1937, leg. Mancini (MCSNV); 1 ex., M. Baldo, Caval di Novezza, 1400 m, 4.VII.1971 (cZan); 1 ex., Merano (NHMW). Piemonte: 1 ex., Baldissero Canavese (TO), Monti Pelati, 500 m, 12.III.1989, leg. Giachino (DEI); 3 ex., Leini (TO), 20.-21.II.1965, leg. Osella (cZan); 1 ex., Pedomonte (UR), 6.IV.1971, leg. Zanetti (cZan); 1 ex., S San Sebastiano Curone, Dernice (AL), 22.I.1994, leg. Diotti (MCSNV); 2 exs., Laux (TO) [4502N, 701E], VII.1937, leg. Burlini (MCSNV); 1 ex., Sommariva B. (CN), 4.IV.1982, leg. Aprá (cAng); 1 ex., Bardonecchia (NHMW); 1 ex., San Christoforo (NHMW). Aosta: 1 ex., Pré-St.-Didier, VII.1938, leg. Burlini (MCSNV). Lombardia: 1 ex., Pso. Croce Domini, 1900 m, 28.VI.1993, leg. Assing (cAss); 4 exs., Borgo Ticino (PV), 8.I.1997, leg. Diotti (cAng); 3 exs., Sondrio, Prati ti V. Valeriana, 13.III.1967, leg. Dioli 
(cZan); 1 ex., Sondrio, 30.IV.1974, leg. Dioli (cZan); 2 exs., M. Rolla (SO), 2000 m, 20.\&25.VI.1972, leg. Dioli (cZan); 1 ex., M. Rolla (SO), Mossini, 19.III.1972, leg. Dioli (cZan); 3 exs., Triangia (SO), 20.III.\&7.IV.1972, leg. Dioli (cZan); 1 ex., Faedo (SO), F. Adda, 15.IX.1968, leg. Dioli (cZan); 1 ex., Faedo (SO), 26.VII.1972, leg. Dioli (cZan); 3 exs., same data, but 20.V.1973 (cZan); 2 exs., Lo (SO), S. Luigi Sazzo, 3.XI.1973, leg. Dioli (cZan); 1 ex., Montagna (SO), 6.IV.1972, leg. Dioli (cZan); 1 ex., Val del Livrio (SO), 1300 m, 7.VI.1972, leg. Dioli (cZan); 2 exs., Poggiridenti (SO), 11.XI.1973, leg. Dioli (cZan); 1 ex., Lovero (SO), 2.IX.1973, leg. Dioli (cZan); 1 ex., V. del Livrio (SO), La Costa, 1500 m, 22.VII.1973, leg. Zanetti \& Dioli (cZan); 1 ex., V. del Livrio (SO), La Costa, 1425 m, 10.VI.1973, leg. Dioli (cZan); 3 exs., V. del Livrio (SO), La Piana, 1500 m, 22.VII.1973, leg. Dioli \& Zanetti (cZan); 2 exs., Castellina (SO), XII.1970, leg. Dioli (cZan); 1 ex., M. Padrio (SO), Trivigno, 18.IX.1971, leg. Dioli (cZan); 1 ex., M. Vespolo (SO), Uberti, 15.VI.1971, leg. Dioli (cZan); 1 ex., M. Vespolo (SO), Scarpalelli, 700 m, 27.VII.1973, leg. Dioli (cZan); 1 ex., M. Vespolo, Le Foppe, 27.V.1973, leg. Dioli (cZan); 1 ex., Teglio, 800 m, 15.V.1972, leg. Dioli (cZan); 3 exs., Mezzanino (PV), 29.IV.1996, leg. Diotti (MCSNV); 2 exs., Merone (CO), 5.I.1998, leg. Dioli (MCSNV); 1 ex., Lugana (BS), 23.III.1974, leg. Zanetti (cZan); 1 ex., Madonna del Frassino (BS), 6.IV.1969, leg. Zanetti (cZan); 1 ex., Anfiteatro Morenico del Garda (BS), Lago di Sovenigo, 2.II.1997, leg. Tagliapetra \& Zanetti (cZan); 1 ex., Mantova, III.1969 (cZan); 2 exs., Ostiglia (MN), Palude del Busatello, 19.IV.1982, leg. Osella \& Zanetti (cZan); 1 ex., Ponti sul Mincio (MN), 27.I.1980, leg. Zanetti (cZan); 1 ex., Castellaro, Lagusello (MN), 2.XII.1990, leg. Zanetti (cZan); 5 exs., Porto Mantov. (MN), Soave, 24.IV.1994, leg. Zanetti (cZan); 1 ex., Milano, 6.III.1938, leg. Magistretti (MCSNV); 8 exs., same data, but 23.X.1936 (MCSNV); 1 ex., Pizzo d' Erna, 19.IV.1946, leg. Magistretti (MCSNV); 4 exs., Borgo Priolo, 5.V.1940, leg. Magistretti (MCSNV); 3 exs., Lambrugo, II.1951, leg. Magistretti (MCSNV); 1 ex., Oltre il Colle (NHMW); 1 ex., Monte Bisbino, leg. Leonhard (NHMW); 4 exs., Cogno, leg. Krüger (NHMW); 1 ex., Monte Mattoni (BS), leg. Breit (NHMW); 1 ex., Campolaro (BS), leg. Breit (NHMW). Veneto: 1 ex., M. Baldo, Novezzina, 1250 m, 3.V.1970, leg. Zanetti (cZan); 1 ex., Verona, stadio, 11.IV.1969 (cZan); 1 ex., Verona, stadio, 12.IX.1972 (cZan); 6 exs., Verona, stadio, with Lasius fuliginosus, 23.I.1975, leg. Zanetti (cZan); 2 exs., Verona, Adige, 19.X.1995, leg. Zanetti (cZan); 1 ex., Verona, Spiana, 20.II.1994, leg. Zanetti (cZan); 3 exs., Verona, P.to S. Parcenzio [?], 7.III.1973, leg. Zanetti (cZan); 1 ex., S. Michele Ex. (VR), 31.III.1974, leg. Zanetti (cZan); 1 ex., Rocca del Garda (VR), 20.IV.1984, leg. Zanetti (cZan); 1 ex., Dolce (VR), 1.X.1999, leg. Manfrin (cZan); 1 ex., Cavaion (VR), Palude Canova, 13.III.1994, leg. Zanetti (cZan); 1 ex., Pescantina (VR), V.1995, leg. Zanetti (cZan); 1 ex., V. Lagarina, Brentino (VR), 9.III.1975, leg. Zanetti (cZan); 1 ex., Ossenigo (VR), 24.V.1970, leg. Zanetti (cZan); 1 ex., Isola della Scala (VR), 12.V.1977, leg. Zanetti (cZan); 10 exs., Isola della Scala, Pellegrina (VR), 3.II.1980, leg. Zanetti (cZan); 4 exs., S. Floriano (VR) (cZan); 1 ex., Villafontana (VR), 25.II.1976, leg. Zanetti (cZan); 1 ex., Monti Lessini (VR), Corno Mozzo, 1535 m, 15.VIII.2001, leg. Buzzetti (cZan); 16 exs., Cerea (VR), 17.I.1988, leg. Zanetti (cZan); 1 ex., Fumane (VR), Cascate di Molina, 20.IV.1974, leg. Zanetti (cZan); 1 ex., Parona (VR), 8.VI.1970, leg. Zanetti (cZan); 1 ex., P.te di Veia (VR), 12.XI.1973, leg. Zanetti (cZan); 1 ex., S. Bartolo d. M. (VR), 800 m, 12.XI.1972, leg. Zanetti (cZan); 2 exs., S. Giovanni Lup. (VR), 2.II.1975, leg. Zanetti (cZan); 3 exs., S. Massimo (VR), 24.III.1970, leg. Zanetti (cZan); 2 exs., S. Massimo, 28.III.1969 (cZan); 1 ex., S. Massimo, 2.IV.1971 (cZan); 1 ex., S. Massimo, 24.III.1970 (cZan); 1 ex., M. Tondo (VR), 780 m, 6.I.1972, leg. Zanetti (cZan); 2 exs., Custoza (VR), Val Molini, 24.XI.1974, leg. Zanetti (cZan); 1 ex., Custoza (VR), 22.I.1972, leg. Zanetti (cZan); 2 exs., Chiampo (VI), III.2001, leg. Buzzetti (cZan); 1 ex., Recoaro (VI), 1200 m, 21.V.1972, leg. Zanetti (cZan); 3 exs., Feltre (BL), X.1972, leg. Dioli (cZan); 4 exs., Padova, Stanga, 19.III.1971, leg. Zanetti (cZan); 6 exs., Padova, 1.IV.1971 (cZan); 1 ex., Montegrotto (PD), 5.III.1972, leg. Zanetti (cZan); 3 exs., Roncaglia (PD), P.te S. Nicolo, 14.\&19.III.1972, leg. Dioli (cZan); 3 exs., Abano (PD), S. Daniele, 13.III.1972, leg. Dioli (cZan); 2 ex., Abano, 28.II.1973 (cZan); 2 exs., Camin (PD), 23.II.1973 (cZan); 3 exs., Arqua Petrarca (PD), 17.X.1995, leg. Zanetti (cZan); 2 ex., Montemerlo (PD), 20.I.1974, leg. Zanetti (cZan); 1 ex., S. Lazzaro (PD), 2.XI.1972 (cZan); 2 exs., Padova, 20.I.1970 (cZan); 1 ex., Padova, 19.V.1970, leg. Zanetti (cZan); 1 ex., Padova, 23.II.1075 (cZan); 3 exs., Padova, X.1934, leg. Burlini (MCSNV); 1 ex., Casacorba (TV), XI.1935, leg. Burlini (MCSNV); 1 ex., same data, but X.1938 (MCSNV); 1 ex., Montello (TV), III.1943, leg. Burlini (MCSNV); 1 ex., Ponzano (TV), leg. Burlini (MCSNV); 1 ex., San Pelagio (TV), VI.1951, leg. Burlini (MCSNV); 1 ex., Lovadina 
(TV), IV.1952, leg. Burlini (MCSNV); 7 exs., Colli Euganei, IV.1921, leg. Moczarski \& Scheerpeltz (NHMW). Friuli-Venezia Giulia: 1 ex., Cornino, Tagliamento flood-plain forest, $46^{\circ} 13 \mathrm{~N}, 13^{\circ} 01 \mathrm{E}, 50 \mathrm{~m}$, 12.IX.1998, leg. Schülke (cSch); 1 ex., Cordenons (PN), Fiume Meduno, V.1967 (cZan); 1 ex., Pordenone, V.1966 (cZan); 1 ex., Lago di Grado, IX.1978, leg. Dioli (cZan); 6 exs., Treporti (VE), VII.1995, leg. Zanetti (cZan); 1 ex., Portegrandi (VE), XI.1939, leg. Burlini (MCSNV); 2 exs., Casarsa, IV.1947, leg. Burlini (MCSNV); 1 ex., Anduins, 13.IV.1936, leg. Burlini (MCSNV); 2 exs., M. Arghena, Prescudin, 1975, Molinieti (cZan); (cZan); 1 ex., Basovizza, IV.1921, leg. Moczarski \& Scheerpeltz (NHMW). Liguria: 6 exs., ca. 10 km NE Genova, Fontanegli, IX.1916, Baliani (MCSNV); 6 exs., same data, but 1917 (MCSNV). Emilia Romagna: 1 ex., 3 km N Castiglione dei Pépoli, ca. 700 m, 3.V.1998, leg. Wolf (cSch); 3 exs., Salsomaggiore (PR), 19.XI.1972, leg. Dioli (cZan); 1 ex., Sarsina, 15.IV.1976 (cZan); 1 ex., Berceto, 21.IV.1939, leg. Magistretti (MCSNV); 1 ex., Lagdei, 1.VI.1948, leg. Magistretti (MCSNV); 1 ex., Trevorzo (PC), 3.V.1996, leg. Diotti (cAng); 1 ex., Passo Pelizzone (PC), 800 m, 10.X.1996, leg. Zanetti (cZan). Toscana: 1 ex., Figline Valdarno (FI), 14.VI.1992, leg. Assing (cAss); 1 ex., Monti della Calvana, Mangona [ca. $44^{\circ} 02 \mathrm{~N}, 11^{\circ} 11 \mathrm{E}$ ], $650 \mathrm{~m}, 1 . V .1991$, leg. Wunderle (cWun); 1 ex., Vallombrosa, Mte. Secchieta, $1350 \mathrm{~m}$, 28.IV.1991, leg. Wunderle (cWun); 1 ex., Orbetello, 3.IV.1974, leg. Vigna (cZan); 1 ex., M. Morello (FI), X.1936, leg. Gagliardi (MCSNV); 1 ex., M. Lombardi (FI), IV.1922 (MCSNV); 1 ex., Pisa, 10.IV.1937, leg. Magistretti (MCSNV); 1 ex., Bientina (PI), V.1935, leg. Gagliardi (MCSNV); 2 exs., Torre del Lago Puccini, IX.1937, leg. Gagliardi (MCSNV); 1 ex., Maresca, 12.VI.1941, leg. Magistretti (MCSNV); 2 exs., Livorno, 28.III.1886, leg. Stöcklin (NHMW). Abruzzi: 1 ex., L'Aquila, leg. Reitter (DEI); 1 ex., L'Aquila (NHMW); 1 ex., Pescasseroli, 1200 m, VIII.1975, leg. Dioli (cZan); 6 exs., Cerchio (NHMW). Marche: 1 ex., M. Sibillini (AP), Gola d' Infernaccio, 700 m, 2.VII.2002, leg. Zanetti (cZan); 1 ex., Gran Sasso, M. Cristo (AQ), 1500 m, 4.V.2000, leg. Angelini (cAng). Lazio: 1 ex., Latina, Monte Circeo, 29.XII.1994, leg. Assing (cAss); 2 exs., Camerata Nuova, 1909, leg. Krüger (DEI); 2 ex., Roma, Colle del Forno, 11.I.1974, leg. Rossi (cZan); 1 ex., same locality, 24.XI.1974, leg. Rossi (cZan); 4 exs., Roma, near Montelibretti, 15.IV.1975, leg. Rossi (cZan); 1 ex., Roma, Ardea, 8.V.1975, leg. Rossi (cZan); 1 ex., Roma, Ciciliano, 1997, leg. Latella (cZan). Campania: 20 exs., estuary of Volturno river (CE), detritus, 25.III.1994, leg. Angelini (cAng, cAss; 1 ex., Centola (SA), S. Severino, III.1986, leg. Dioli (cZan). Puglia: 1 ex., Daunia, Volturino (FG), 750 m, 12.V.2001, leg. Angelini (cAss). Basilicata: 1 ex., Oasi WWF "La San Giuliano", Ponte Cagnolino, 21.III.1993, leg. Anglini (cAss); 1 ex., Oasi WWF "Lago Pantano di Pignola" (PZ), 770 m, 30.V.1991, leg. Angelini (cAng). Locality ambiguous: 1 ex., Varano, 1899, leg. Paganetti (DEI). Locality not specified: 3 exs., "Ital." (NHMW).

\section{Remarks}

The species is widespread in Italy and has been recorded southwards to Basilicata and Puglia (Map 1). It is apparently much rarer in the south, where $D$. italica and $D$. angelinii occur, than in the northern and central parts of mainland Italy. This conclusion is not based on biassed collecting activity, since the south of the Italian mainland has been extensively studied by the coleopterist F. Angelini. In the course of the present study, no material from Calabria and from the islands was seen. Specimens from Sicily identified as D. canaliculata all proved to refer to D. italica. Luigioni (1929) reports D. canaliculata from Sardinia and Sicily, but these records are apparently based on misidentifications (see also ZanetTi 1995). For observations on the life history (larval stages, phenology) and other bionomic data see Assing (1994) and Schminke (1982).

\section{Drusilla memnonia (MärKEL, 1845) (Map 1)}

Material examined [for more examined material from Tunisia see Assing (2005c)]: Sicilia: 1 ex., Madonie, Piano Bottaglia, 13.III.1970 (cAss); 1 ex., Piano Bottaglia, $31 . I I I .1970$ (cAss); 3 exs., Cotano, Castelluzzo (TP), C. da Calazza, 2.III.1994, leg. Sabella (cAss); 1 ex., Castelvetrano (TP), V.ne Zangara, 11.III.1993, leg. Sabella (cAss); 3 exs., Erice (TP), mixed forest, 10.XII.1992, leg. Sabella (cAss); 1 ex., Petralia, 8.XII.1975, 
leg. Curti (cSch); 1 ex., Caltagirone, 15.XII.1975, leg. Curti (cSch); 2 exs., Ficuzza, 16.III.1942 (DEI); 2 exs., Ficuzza, 18.IV.1942 (NHMW); 12 exs., Ficuzza, 1906, leg. Leonhard (DEI, NHMW); 8 exs., Palermo (DEI, NHMW); 20 exs., locality not specified (DEI, NHMW).

Algeria: 4 exs., "Bona” (DEI); 7 exs., "Algier" (DEI). Tunisia: 2 exs., Le Kef (DEI); 5 exs., “Tunis" (DEI).

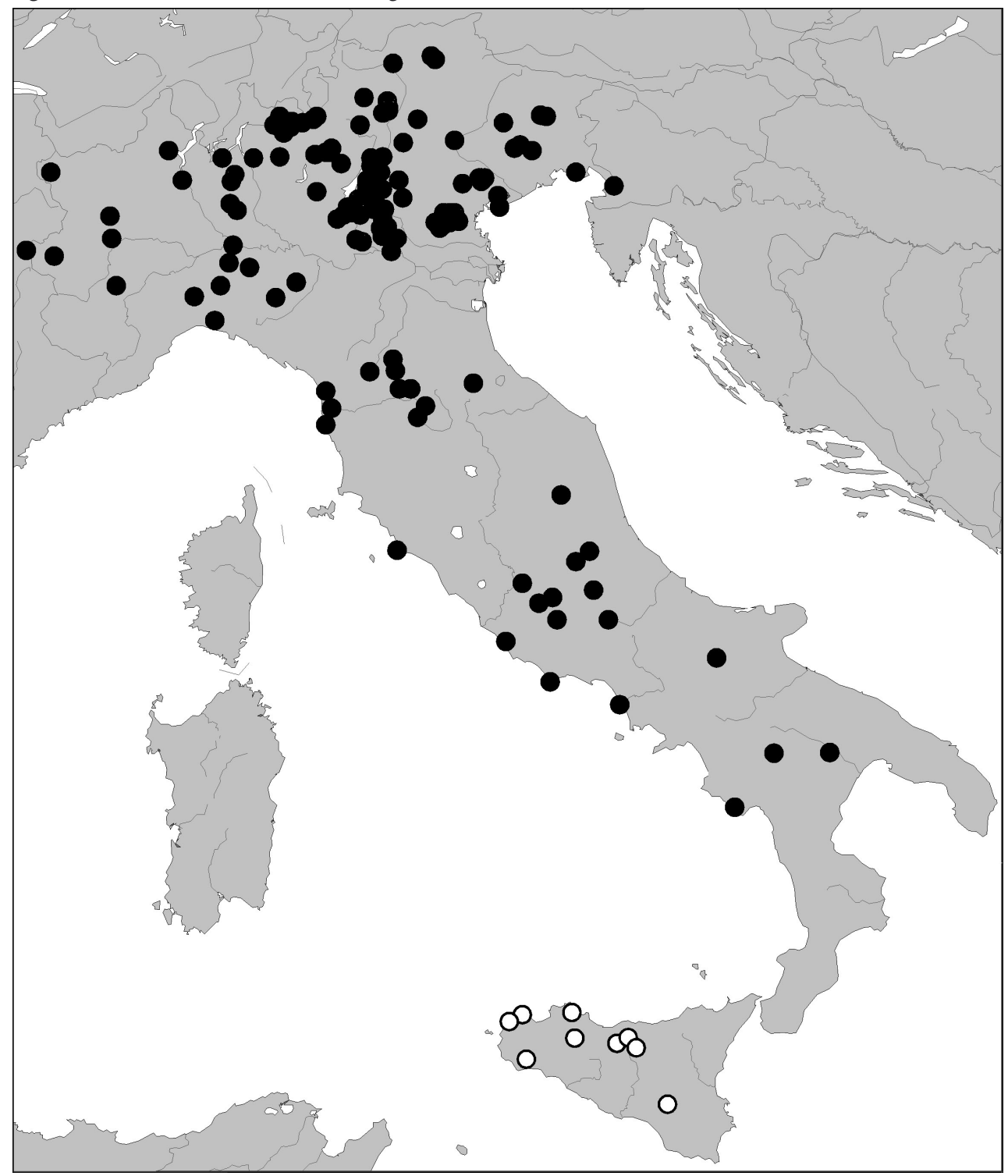

Map 1: Distributions of Drusilla canaliculata (filled circles) and D. memnonia (open circles) in Italy, based on examined records.

\section{Remarks}

In Italy, the distribution of this species is confined to Sicily (Map 2). It has also been recorded from Tunisia and Algeria; for more details see Assing (2005c). PorTA (1926) indicates $D$. memnonia from Sardinia, but this record is apparently based on a misidentification (see also ZanetTi 1995). 


\section{Drusilla italica (BERnhauer, 1903) (Figs 7-52, Map 2)}

\section{Type material}

Lectotype 우 [dissected prior to present study, spermatheca damaged]: Vallo Lucania, Süditalien, Solari / meridionalis Fauvel / italicus Bernh., Type / Chicago NHMus., M.Bernhauer Collection / Drusilla italica (Bh.) V.I. Gusarov det. 1993 / Lectotypus 우 Astilbus italicus Bernhauer desig. V. Assing 2006 / Drusilla italica (Bernhauer) det. V. Assing 2006 (FMNH).

\section{Additional material examined:}

Form 1 : Campania: 2 exs., Vallo Lucano, San Biase, 1904, leg. Solari (NHMW, cAss). Calabria: 22 exs., Sant'Eufemia d'Aspromonte, fiumara Crasta, 650 m, 4.V.1993, leg. Angelini \& Sabella (cAss); 2 exs., Calabria, Sant'Eufemia d'Aspromonte, fiumara Crasta, 6.V.1993, chestnut forest, leg. Angelini \& Sabella (MCSNV); 3 exs., same locality, 550 m, 31.V.1993, leg. Angelini (MCSNV); 1 ex., same locality, 630 m, 31.V.1993, leg. Angelini \& Sabella (cAng); 7 exs., same locality, 600 m, 23.VI.1993, leg. Angelini (cAng); 5 exs., below S. Eufemia-Piani Aspromonte, 800 m, Quercus ilex forest, 21.VI.1993, leg. Angelini (MCSNV); 2 exs., same locality, 650 m, chestnut forest, 31.V.1993, leg. Angelini \& Sabella (cAng); 1 ex., same locality, 1905, leg. Paganetti (DEI); 8 exs., same locality, leg. Paganetti (DEI, NHMW); 1 ex., same locality, VII.1957 (MCSNV); 5 exs., Caraffa del Bianco (RC), M. Mena, grassland, 14.IV.1997, leg. Angelini (cAss); 3 exs., Africo (RC), grassland, 50 m, 7.XI.1997, leg. Angelini (cAss); 1 ex., Africo (RC), 50 m, grassland, 23.I.2003, leg. Angelini (cAng); 1 ex., same data, but 29.V.2003 (cAng); 8 exs., Femuzzano (RC), 70 m, grassland, 23.I.2003, leg. Angelini (cAss); 11 exs., S. Luca env. (RC), grassland, 8.XI.1997, leg. Angelini (cAss); 1 ex., S. Luca, loc. S. Giorgio, 550 m, stream bank, 13.X.1993, leg. Angelini (MCSNV); 1 ex., San Luca (RC), grassland, 200 m, 23.I.2003, leg. Angelini (cAng); 1 ex., S. Luca, road to Santuario di Polsi, 740 m, Quercus ilex forest, 13.X.1993, leg. Angelini (cAng); 3 exs., S. Alessio (RC), 490 m, oak forest, 2.V.1993, leg. Sabella (cWun); 10 exs. [originally identified as D. canaliculata], S. Alessio, $470 \mathrm{~m}$, chestnut forest, 2.V.1993, leg. Angelini \& Sabella (MCSNV, cAss); 1 ex., Antonimina (RC), 800 m, mixed forest, 3.V.1993, leg. Angelini \& Sabella (cWun); 7 exs., Antonimina, 600 m, Quercus ilex forest, 1.VI.1993, leg. Angelini \& Sabella (MCSNV, cAng); 1 ex., Antonimina, 800 m, Quercus ilex forest, 3.V.1993, leg. Angelini \& Sabella (cAng); 1 ex., same data, but 600 m (cAng); 6 exs., Antonimina, 1905, leg. Paganetti (DEI); 3 exs., Antonimina, leg. Pagenetti (NHMW); 11 exs., Cimina, leg. Paganetti (DEI, NHMW); 2 exs., Cimina, 650 m, Quercus ilex forest, 12.X.1993, leg. Angelini \& Sabella (MCSNV); 7 exs., Gerace, leg. Paganetti (NHMW); 3 exs., Samo (RC), Fiumara S. Venere, Mastix, 7.XI.1997, leg. Angelini (cAng); 3 exs., Samo (RC), Fiumara S. Venere, grassland, 14.IV.1997, leg. Angelini (cAss); 1 ex., Podàrgoni (RC), 600 m, stream, 22.VI.1993, leg. Angelini (cAng); 6 exs., Isola di Capo Rizzuto (KR), 14.XII.1997, leg. Angelini (cAss); 6 exs. [1 teneral], Cetraro, Aron stream (CS), 16.VI.1994, leg. Angelini (cAss); 2 exs., Fagnano Castello [CS], Lago Trifoglietti, 1300 m, VI.1994, Angelini \& Sabella (cAss); 1 ex., Roseto (CS), grassland, 28.XII.1997, leg. Angelini (cAng); 8 exs., Monti Orsomarso, 670 m, 4.VIII.1989, leg. Angelini (cAss, cWun); 3 exs., Monti Orsomarso, Grisolia (CS), loc. Pantanelle, 700 m, beech forest, 17.VI.1997, leg. Angelini (cAss); 5 exs., same locality, 670 m, beechforest, 4.VIII.1989, leg. Angelini (cAng); 3 exs., Monti Orsomarso, Grisolia (CS), 8.VIII.1992, 700 m, beech forest, leg. Angelini (cAng); 3 exs., Monti Orsomarso, Valle Argentino (CS), 250 m, oak forest, 11.VIII.1990, leg. Angelini (cAng); 1 ex., Valle Argentino, oak forest, 16.VI.1991, leg. Angelini (cAng); 1 ex., Monti Orsomarso, S. Maria del Monte (CS), 1350 m, chestnut forest, 6.VIII.1989, leg. Angelini (cAng); 3 exs. [one specimen with worker of Formica sanguinea attached to the pin], Sila Grande (CS), Lago Arvo, south shore, 1290-1350 m, 18.-19.V.1994, leg. Schulz, Güsten \& Sanetra (cAss); 3 exs., E Cittanova, Piani di Gióia, Pso. del Mercante, 500 m, 30.VI.2001, leg. Wolf (cSch, cAss). Basilicata: 1 ex., Monte Sirino (PZ), 1300 m, 26.VI.1998, leg. Daccordi (cAss); 5 exs., M. Sirino, Timpa Pellinera (PZ), 900 m, beech and oak forest, 30.VII.1989, leg. Angelini (cAng); 1 ex. [teneral], M. Sirino, L. Sirino env. (PZ), 800 m, oak forest, 25.VII.1989, leg. Angelini (cAng); 1 ex., Lagonegro (PZ), ponte La Cala, 600 m, 6.VIII.1990, leg. Angelini (cZan); 3 exs., Episcopia (PZ), 520 m, bank of Sinni river, trap with acetic acid, 19.VI.2002, leg. Angelini (cAng, cAss); 1 ex., Abriola, La Maddalena (PZ), 
1400 m, trap with acetic acid, 30.III.2002, leg. Angelini (cAng); 1 ex., Castelsaraceno (PZ), Cogliandrino stream, trap with acetic acid, 800 m, 14.IV.2004, leg. Angelini (cAng); 1 ex., Rivello, Monte Caccovello (PZ), 630 m, oak forest, 29.VII.1989, leg. Angelini (cAss). Puglia: 23 exs., Taranto env., 17.XII.2000, leg. Montemurro (cZan, cAss); 16 exs., Taranto env., 22.X.2000, leg. Montemurro (cZan). Sicilia: 1 ex., Monti Nebrodi, E Portella Femmina Morta, SE-slope, 3755N, 14³9E, 1560 m, 2.IV.2001, leg. Schülke (cSch); 1 ex., Messina, 1906, leg. Leonhard (cAss); 2 ex., Messina, 26.IV.1943 (NHMW); 1 ex., Ficuzza, 1906, leg. Leonhard (cAss); 2 exs., Fiumara di Niceto, 1.IX.1995, leg. Adorno (cZan).

Form 2: Abruzzi: 1 ex., Aquila (NHMW). Lazio: 2 exs., Camerata Nuova, 1909, leg. Krüger (NHMW, cAss); 1 ex., Itri, 25.IV.1977, leg. Zampetti (cAss). Campania: 17 exs., estuary of Volturno river (CE), detritus, 25.III.1994, leg. Angelini (cAng, cAss); 10 exs., Salerno, Com. Capaccio, E Paestum, west side of Mte. Soprano, 350 m, 13.X.2000, leg. Wolf (cAss, cSch); 1 ex., Rofrano (SA), 400 m, 11.IV.2004, leg. Zanetti (cZan); 1 ex., Cilento, M. Cervati (SA), 1100 m, beech forest, 31.VII.1989, leg. Angelini (cAng). Basilicata: 3 exs., Policoro (MT), 14.IX.1999, leg. Angelini (cAss); 1 ex., Policoro (MT), 25.I.1976, leg. Angelini (cWun); 1 ex., Policoro (MT), 23.IV.1978, leg. Angelini (cZan); 1 ex., Policoro, 4.I.1976, leg. Angelini (cAng); 1 ex., Policoro, 24.VII.1994, leg. Angelini (cAng); 1 ex., Policoro, 2.VI.1989, leg. Angelini (cAng); 2 exs., Valsinni (MT), 180-200 m, rive F. Sinni, 13.X.2002, leg. Angelini (cAng); 1 ex., same data, but 19.VI.2002 (cAng); 1 ex., Potenza, X.1995, leg. de Marzo (cAss); 1 ex., Pietrapertosa, M. Impiso (PZ), 1200 m, 6.VII.1989, leg. Angelini (cAss); 2 exs., Accettura, torrente Salandrella (MT), 425 m, oak forest, 14.V.1989, leg. Angelini (cAng, cAss); 3 exs., Laghi di Monticchio (PZ), 660 m, 19.VI.1988, leg. Angelini (cAng); 1 ex., road Lago Pantano-La Sellata (PZ), 950 m, 30.VI.2002, leg. Angelini (cAng); 3 exs., same data, but 27.VII.2002 (cAss); 1 ex., same data, but 900 m, 14.IV.2002 (cAng); 1 ex., Accettura, Monte la Cróccia (MT), 1000 m, oak forest, 1.V.1990, leg. Angelini (cAng); 2 ex., Pollino, San Severino (PZ), bosco Magnono, 800 m, beech forest, 19.XII.1993, leg. Angelini (cAng); 3 exs., Corleto Perticara (PZ), 770 m, 1.VII.1989, leg. Angelini (cAng); 1 ex., Corleto Perticara, 970 m, 14.V.1989, leg. Angelini (cAng); 1 ex., L. S. Giuliano (MT), Ponte Cagnolino, 18.VIII.1993, leg. Angelini (cAng). Puglia: 1 ex., L. San Giovanni, Gargano, leg. Holdhaus (cAss); 4 exs., F. Lato, 10 km from estuary (TA), 2.I.1977, leg. Angelini (cZan, cAss); 1 ex., 5 km E Taranto, Circummarpiccolo (TA), 5.XII.1993, leg. Angelini (cAss); 1 ex., Gravina di Puglia (BA), 400 m, 21.IV.1987, leg. de Marzo (cAng); 1 ex., estuary of Bradano river (TA), 15.II.1985, leg. Angelini (cAng); 1 ex., San Basilio (TA) ["Murgien, San Basilio"], leg. Paganetti (NHMW); 1 ex., Taranto, 21.VII.1956, leg. Viglietta (cWun). Locality not specified: 1 ex.: Italia (DEI).

\section{Redescription}

4.0-5.5 $\mathrm{mm}$. Coloration dark brown to blackish, pronotum and elytra often slightly to distinctly paler than head and abdomen; legs yellowish to pale brown; antenna brown to dark brown, with the basal 3-4 antennomeres reddish to reddish brown.

Head with posterior angles more or less marked to smoothly rounded; puncturation distinct, but rather fine and shallow, moderately dense; median dorsal area sometimes punctate; interstices in lateral dorsal area narrower than diameter of punctures; microsculpture variable, ranging from shallow and indistinct to pronounced (Figs 7-8, 21-24). Antenna of variable length and proportions (Figs 41-43).

Pronotum with very dense puncturation; punctures denser and more distinct than those of head; interstices with variable, shallow to distinct, but occasionally practically obsolete microsculpture, and with weak to distinct shine; fine furrow along midline not reaching anterior and posterior margins; sexual dimorphism less pronounced than in $D$. canaliculata (Figs 7-8, 21-24).

Elytra 0.55-0.60 times as long as pronotum; laterally more or less extensively and shallowly impressed; puncturation much coarser than that of pronotum; interstices very narrow and with some shine (Figs 7-8, 21-24). 


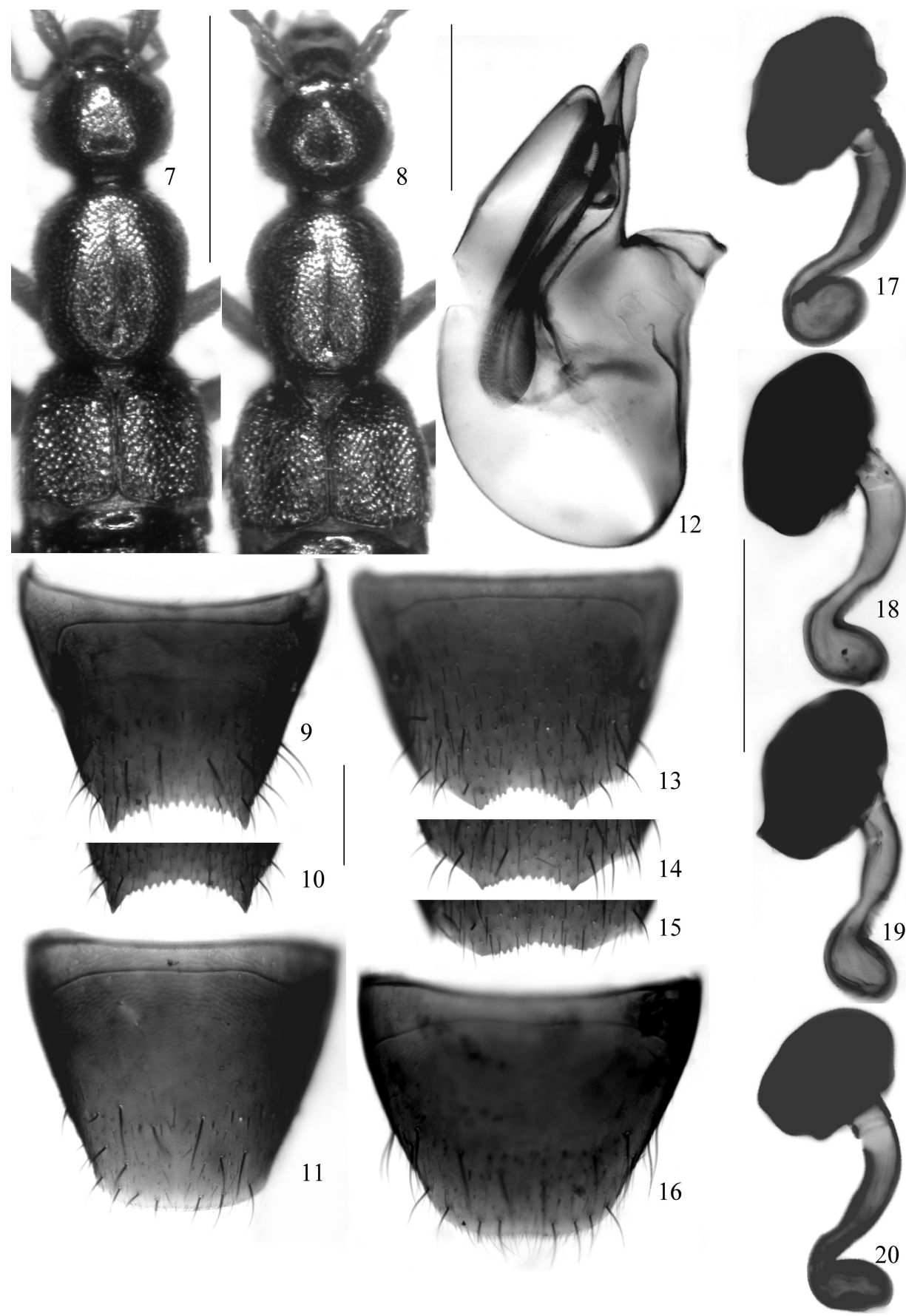

Figs 7-20: Drusilla italica, form 2: o forebody (7); ㅇ forebody (8); o tergite VIII (9); posterior margin of $\sigma^{\star}$ tergite VIII (10); $\sigma^{\star}$ sternite VIII (11); median lobe of aedeagus in lateral view (12); $\sigma^{\star}$ tergite VIII (13); posterior margin of ㅇ tergite VIII (14-15); ㅇ sternite VIII (16); spermatheca (17-20). Scale bars: 7-8: $1.0 \mathrm{~mm}$; 9-20: $0.2 \mathrm{~mm}$. 


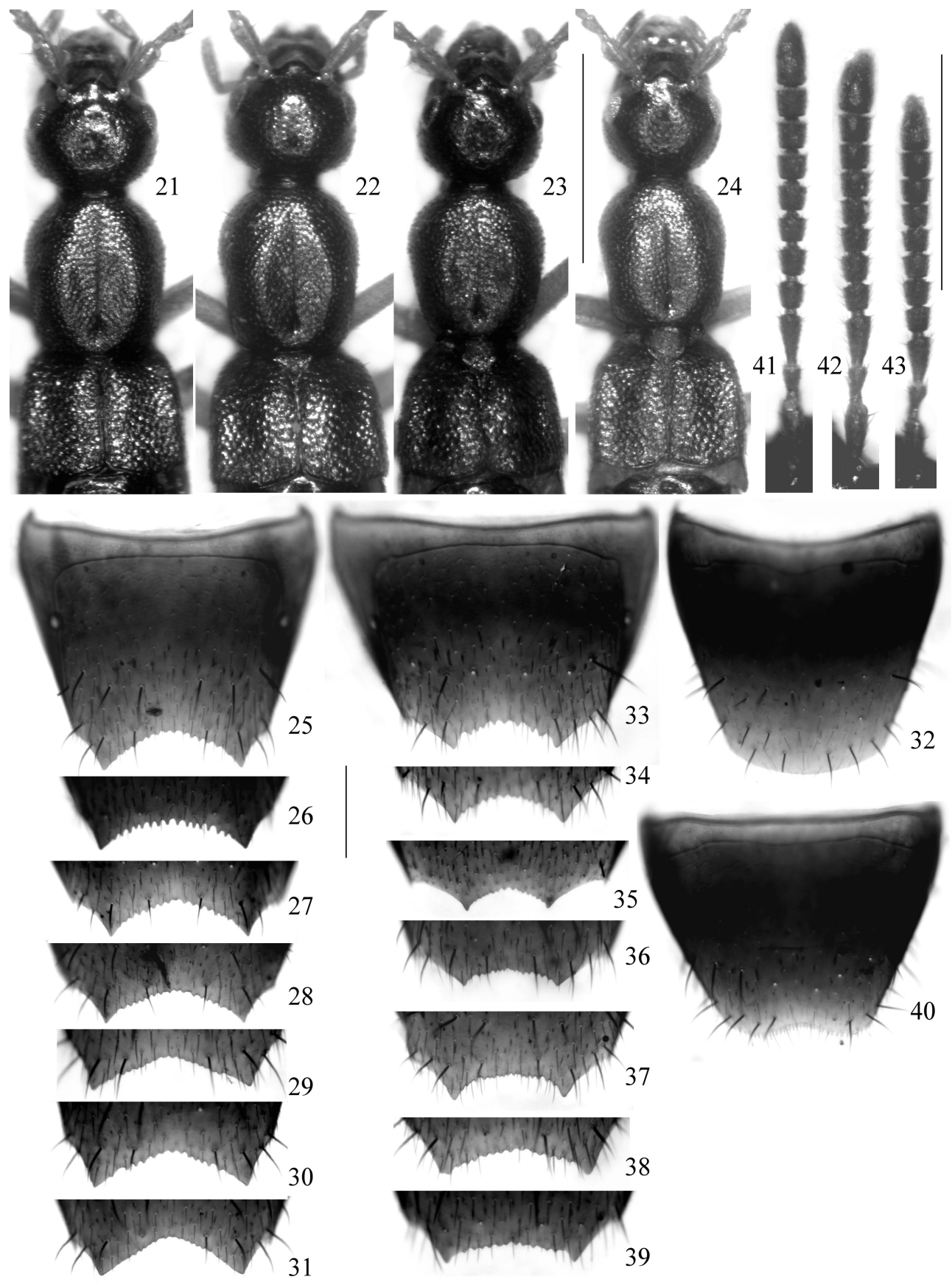

Figs 21-43: Drusilla italica, form 1: o forebody (21-23); o forebody (24); $0^{\star}$ tergite VIII (25); posterior

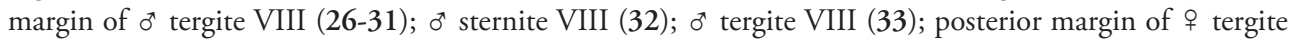
VIII (34-39); + sternite VIII (40); antenna (41-43). Scale bars: 21-24, 41-43: $1.0 \mathrm{~mm}$; 25-40: $0.2 \mathrm{~mm}$. 

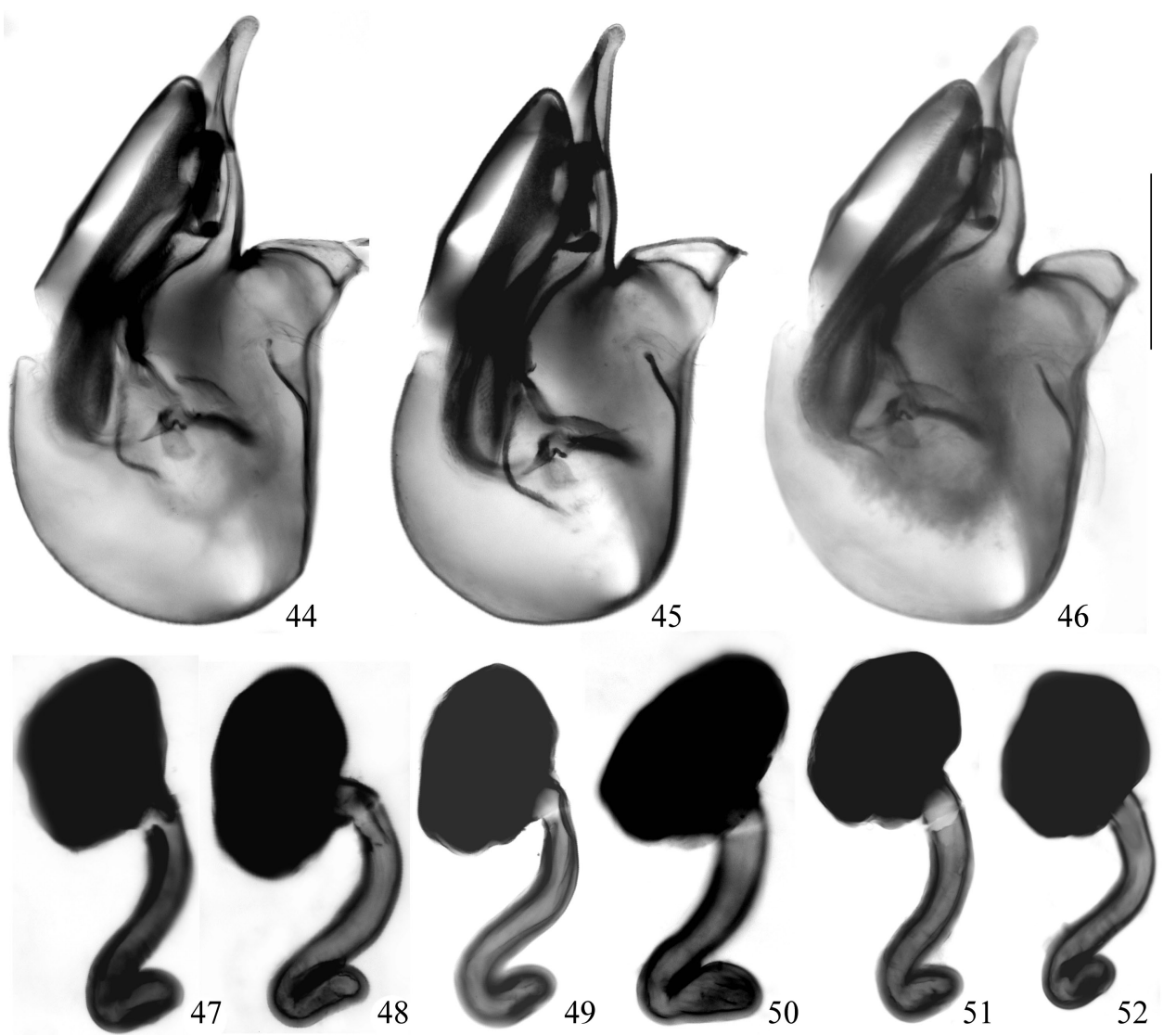

Figs 44-52: Drusilla italica, form 1: median lobe of aedeagus in lateral view (44-46); spermatheca (47-52). Scale bars: $0.2 \mathrm{~mm}$.

Abdomen without or with very superficial microsculpture; puncturation moderately dense on anterior tergites and very sparse on posterior tergites; posterior margin of tergite VII with barely noticeable rudiment of a palisade fringe.

$\sigma^{*}$ : posterior two thirds of pronotum with median impression of variable depth (but always shallower and less extensive than in D. canaliculata) (Figs 7, 21-23); posterior margin of tergite VIII serrate and broadly concave, this concavity of variable depth, often somewhat angulate in the middle, and laterally often delimited by pronounced tooth-like process (Figs 9-10, 25-31); sternite VIII strongly tapering posteriorly, its hind margin weakly convex (Figs 11,32); median lobe of aedeagus rather uniform (Figs 12, 44-46).

9: posterior half of pronotum with shallower median impression (Figs 8, 24); posterior margin of tergite VIII weakly serrate and more narrowly concave than in $0^{\text {t }}$, this concavity of variable depth (Figs 13-15, 33-39); posterior margin of sternite VIII usually very shallowly concave (Figs 16, 40); spermatheca of rather variable shape, with strongly sclerotised, usually almost black capsule and with subproximally often narrowed and proximally more or less distinctly dilated duct (Figs 17-20, 47-52). 


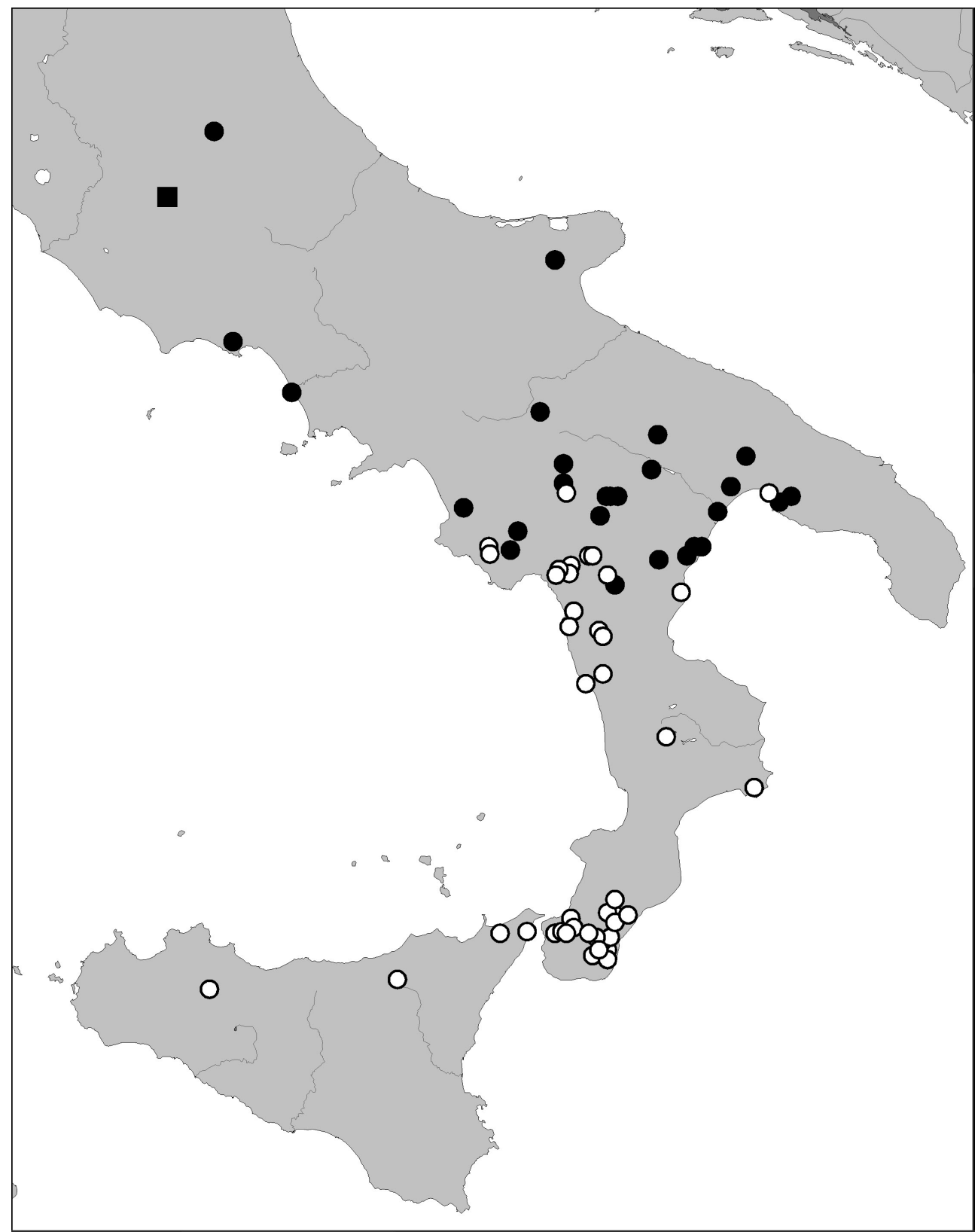

Map 2: Distribution of Drusilla italica (open circles: form 1; filled circles: form 2; sqare: specimens from Camerata Nuova) in southern Italy, based on examined records.

\section{Intraspecific variation}

External characters such as size, coloration, microsculpture, and pronotum shape, the secondary sexual modifications of the pronotum and the abdominal tergite VIII, as well as the morphology of the spermatheca were observed to be subject to pronounced varia- 
tion. This observation at first suggested that D. italica was in fact a complex of two or more species or subspecies, particularly because the spermatheca is usually of rather constant shape and of high taxonomic significance in the genus (Assing 2005b). Based on a systematic and comparative study of these characters and a zoogeographic analysis, several forms or character constellations, as well as zoogeographic trends were identified:

Specimens from the extreme south of mainland Italy (from southern Puglia and southern Campania to Calabria) and from Sicily (Map 2) - hereafter referred to as form 1 - tend to have a distinctly microsculptured head and pronotum, usually paler, light to dark brown pronotum and elytra, a usually more slender and more convex (cross-section) pronotum (Figs 21-24), weakly marked posterior angles of the head, and a spermatheca with a proximally variable, but generally rather weakly dilated and subproximally not distinctly narrowed duct (Figs 47-52). Material from Sicily is characterised by larger average size than beetles from mainland Italy, which is one reason why they were occasionally misidentified as $D$. canaliculata in the collections examined. The lectotype of $D$. italica belongs to form 1.

Form 2 is characterised by a more shiny appearance due to shallower microsculpture of head and pronotum, more uniform dark coloration (pronotum and elytra usually as blackish as head and abdomen), a usually slightly shorter and less convex pronotum (Figs 7-8), and a spermatheca with a proximally distinctly dilated and subproximally often constricted duct (Figs 17-20). This character constellation was often observed in material from the region comprising northern Campania, Lazio, Abruzzi, Basilicata, and Puglia (Map 2).

In some regions such as Campania, Basilicata, and southeastern Puglia, both forms occur. Also, transitional character states were observed not only for the external characters, but also for the shape of the spermatheca, which is quite variable also within the populations here referred to as form 2 (Figs 47-52). Finally, two specimens seen from Camerata Nuova (Lazio) - in the north of the range of form 2 (see square in Map 2) - have the external characters typically found in form 1, but a spermatheca similar to that of form 2. If the two forms represented subspecies, such an intermediate character constellation should be expected in the areas where both distributions overlap, but not in the north of the range of form 2. Consequently, in view of the distribution of morphological characters outlined above and the zoogeographic data, the observed variation is attributed to clinal rather than (sub-)specific variation.

\section{Distribution and bionomics}

The distribution of this species is confined to the south of mainland Italy and Sicily, with the northernmost records from Lazio, Abruzzi, and the Monte Gargano in northern Puglia (Map 2). Records from northern Italy (Luigioni 1929, PorTa 1926, ZanetTi 1995) are doubtlessly based on a confusion with other species. The material examined was collected at altitudes of 50-1560 m, both in pastures and in different types of deciduous forests (oak, beech, chestnut). On one occasion, three specimens were found in a nest of Formica sanguinea LATreIlle. Adult beetles were found throughout the year: January (16 exs./6 records), February (1/1), March (19/3), April (13/7), May (54/15), June (51/16), July (18/10), August (22/7), September (5/2), October (33/7), November (17/3), December (33/5). Teneral adults were collected in June and July. 


\section{Drusilla erichsoni (PeYRon, 1857)}

\section{Remarks}

The records of D. italica from the Cottian Alps (Luigioni 1929) and from northern Italy (ZANETTi 1995) may refer to $D$. erichsoni, of which I have seen material only from southeastern France (Assing 2005b). The presence of this species in Italy is doubtful and requires verification.

\section{Key to the Italian species of Drusilla}

Apart from the three species whose presence in Italy has been verified, the following key also accounts for $D$. erichsoni, whose presence in northwestern Italy is doubtful, but not unlikely.

1. Dark-coloured species; legs completely or almost completely blackish brown to blackish. Distribution: Italy: Sicily (Map 1); Tunisia; Algeria. D. memnonia

Legs yellowish to pale brown. 2

2. On average larger species. Sexual dimorphism of pronotum pronounced, in $\sigma^{\star}$ deeply and extensively impressed (Figs 1-2). $\sigma^{\star}$ : posterior margin of tergite VIII very broadly concave (Fig. 3); sternite VIII weakly tapering posteriad, its posterior margin almost truncate; aedeagus larger and with ventral process apically acute in lateral view (Fig. 4). + : posterior margin of tergite VIII broadly and weakly concave (Fig. 5); spermatheca with slender, weakly sclerotised capsule and with relatively long, almost S-shaped duct (Fig. 6). Widespread in Palaearctic region, introduced in North America; in Italy confirmed records only from the mainland (Map 1).

D. canaliculata

- On average smaller species. Sexual dimorphism of pronotum less pronounced, in $\sigma^{\star}$ more shallowly and less extensively impressed. $\sigma^{\star}$ : posterior margin of tergite VIII more narrowly concave; sternite VIII distinctly tapering posteriad; aedeagus smaller and with ventral process apically less acute in lateral view. + : posterior margin of tergite VIII with less broad concavity; spermatheca with large, strongly sclerotised, almost black capsule, and with short duct of different shape. 3

3. Head and pronotum with pronounced shine, usually without, at most only with indistinct traces of microsculpture (Fig. 13 in Assing 2005b); puncturation of head and pronotum sparser and on average finer, interstices on pronotum wider than diameter of punctures. o : spermatheca with oval (i. e. weakly oblong) capsule in lateral view; proximal part of spermathecal duct longer (Fig. 18 in Assing 2005b). Species from southeastern France, possibly occurring also in northwestern Italy. D. erichsoni

Head and pronotum with subdued shine due to denser puncturation and at least shallow microsculpture; interstices on pronotum narrower than punctures (Figs 7-8, 21-24). ㅇ: spermatheca with transverse capsule; proximal part of spermathecal duct short (Figs 17-20, 47-52). Species from southern Italy, northwards to Lazio, Abruzzi, and northern Puglia (Map 2). D. italica 
On Drusilla taygetana Assing (Figs 53-55)

The original description of this recently described species is based on a single female holotype. In the course of the present paper, a male from the Taygetos (Greece: Pelopónnisos) was found among the Drusilla material from the collections of the DEI, so that the male primary and secondary sexual characters can now be described:

Pronotum deeply and extensively impressed (more so than in the sympatric D. meridiana) (Fig. 53); tergite VIII with broader posterior emargination than in D. meridiana (Fig. 54); median lobe of aedeagus larger than in D. meridiana (Fig. 55).
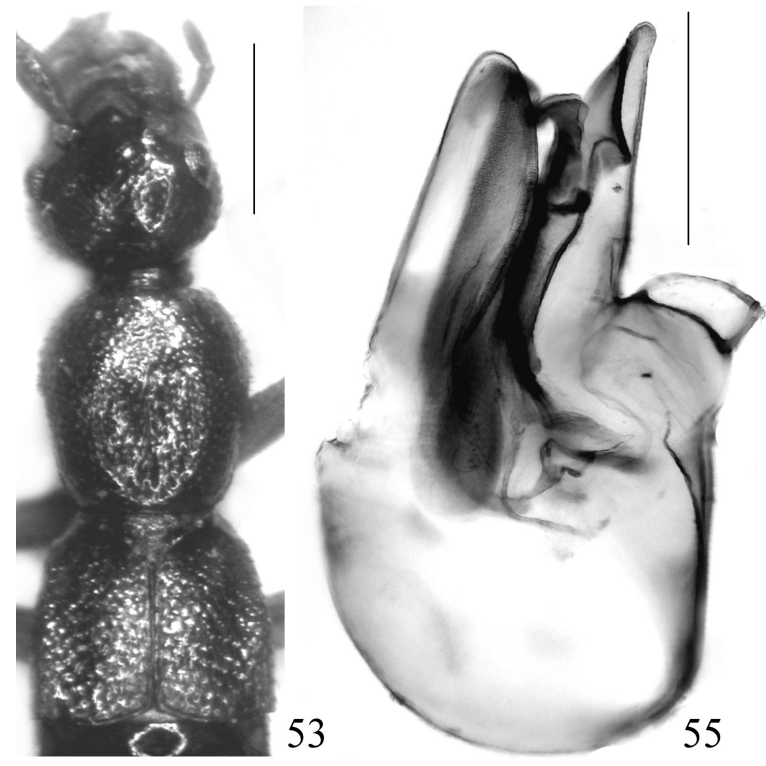

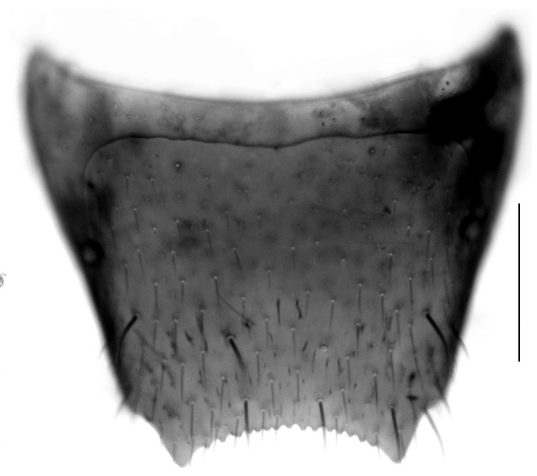

54

Figs 53-55: Drusilla taygetana: forebody (53); o tergite VIII (54); median lobe of aedeagus in lateral view (55). Scale bars: 53: $0.5 \mathrm{~mm}$; 54-55: $0.2 \mathrm{~mm}$.

\section{Acknowledgements}

I am grateful to all the colleagues indicated in the material section for the loan and gift of material under their care.

\section{References}

Assing, V. 1994: Zur Kurzflügelkäferfauna xerothermer Flächen im südlichen Niedersachsen (Coleoptera: Staphylinidae). - Göttinger naturkundliche Schriften 3: 7-31.

Assing, V. 2005a: A revision of the Middle Asian species of Drusilla Leach (Insecta: Coleoptera: Staphylinidae: Aleocharinae) - Entomologische Blätter 101: 43-56.

Assing, V. 2005b: On the western Palaearctic species of Drusilla LEACH, with special reference to the species of the eastern Mediterranean (Coleoptera: Staphylinidae, Aleocharinae). - Koleopterologische Rundschau 75: 111-149. 
Assing, V. 2005c: New species and records of Staphylinidae from Tunisia (Insecta: Coleoptera). - Linzer biologische Beiträge 37: 749-770.

Bernhauer, M. 1903: Neue Staphyliniden der paläarktischen Fauna. - Münchener Koleopterologische Zeitschrift 1: 187-192.

HLAvÁč, P. 2005: Revision of the myrmecophilous genus Lomechusa (Coleoptera: Staphylinidae: Aleocharinae). - Sociobiology 46: 203-250.

Luigioni, P. 1929: I coleotteri d'Italia. Catalogo sinonimico-topografico-bibliografico. - Memorie della Pontificia Accademia delle Scienze (II) 13: 1-1160.

Porta, A. 1926: Fauna Coleopterorum Italica, 2. Staphylinoidea. - Piacenza: 1-405.

Schminke, G. 1982: Larven und Fortpflanzungsverhalten von Drusilla canaliculata, Zyras humeralis, Geostiba circellaris und Othius myrmecophilus (Coleoptera: Staphylinidae). - Drosera 82: 91-100.

Zanetti, A. 1995: Habrocerinae, Trichophyinae, Tachyporinae e Aleocharinae (generi 148-314), pp. 33-58. - In: Ciceroni, A.; Puthz, V. \& Zanetti, A.: Coleoptera, Polyphaga III (Staphylinidae). Checklist delle specie della fauna italiana. - Calderini Bologna, Fasc. 48: 1-65.

\section{Author's address:}

Dr. VolKer Assing

Gabelsbergerstr. 2

D - 30163 Hannover

Germany

e-mail: vassing.hann@t-online.de

\section{Subject editor:}

B. Klausnitzer 